\title{
Adaptive Compensator of Single State Elastoplastic Friction Model
}

\author{
A. A. Abouelsouda, J. Abdo*b and R. Zaierb \\ ${ }^{a}$ Department of Electronics and Communications Engineering, Faculty of Engineering, Cairo University, Egypt \\ ${ }^{*}$ Department of Mechanical and Industerial Engineering, College of Engineering, Sultan Qaboos University, P.O. Box 33, \\ Postal Code 123, Al-Khoud, Muscat, Oman
}

Received 19 April 2011; accepted 25 September 2011

$$
\begin{aligned}
& \text { معوض متوائم للاحتكاك الممثل بنموذج مرن لدن وحيد الحالة }
\end{aligned}
$$

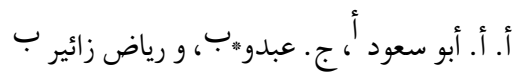

$$
\begin{aligned}
& \text { الخلاصة: ظاهرة الاحتكاك غير الخطي والتي لا يككن تجنبها تظهر دائما في الأنظمة الميكانيكية بين الأسطح المتلامسة. ولقد تم تصميم معوض متوائم } \\
& \text { للاحتكاك الممثل بنموذج مرن - لدن وحيد الحالة يضمن متابعة أي سرعة مرغوبة بوجود قوى الاحتكاك. وقد تح إثبات إنبات استقرار النظام الكلي } \\
& \text { باستخدام دالة ليابونوف. كما وضحت برامج الحماكاة مدى فاعلية النظام المقترح. } \\
& \text { المفردات المفتاحية: معوض متوائم، نموذج مرن-لدن للاحتكاك، طريقة ليابونوف الثانيه }
\end{aligned}
$$

\begin{abstract}
A nonlinear friction is an unavoidable phenomenon frequently experienced in mechanical system between two contact surfaces. An adaptive compensator is designed to achieve tracking of a desired velocity trajectory in the presence of friction force described by a single state elastoplastic friction model. The adaptive compensator includes an adaptive observer and a computed force controller. The closed loop system is shown to be stable using Lyapunov second method. Simulation results show the effectiveness of the proposed compensator.
\end{abstract}

Keywords: Adaptive compensator, Elastoplastic friction model, Lyapunov second method

\section{Introduction}

Friction occurs in all mechanical systems, eg. bearings, transmission, hydraulic and pneumatic cylinders, valves, brakes and wheels. In many engineering applications, frictional contact occurs between machine parts and the characterization of contact behavior becomes an important subject in solving tribological problems such as friction induced vibration wear. Issues related to mechanical sealing, performance and life of machine elements, and thermal are few examples. The pioneering work of Greenwood and Williamson (1966) has been utilized by many researchers (Ibrahim and Rivin 1994; Mulhearn and Samuels 1962; Abdo and Farhang 2005, Seabra and Berthe 1987) as a basic for further extension to obtain contact models for general or specific contact problems for mainly elastic contact. On the other hand, the work of Pullan and Williamson (1972) utilized as a basic model for pure plastic contact. In an attempt to bridge the gap between the pure elastic and pure

*Corresponding author's e-mail: jdabdo@squ.edu.om plastic contact, researchers Halling and Nuri (1972), Greenwood and Tripp (1971), and Abdo and AlYahmadi (2004) developed a wide intermediate range of interest where elastic-plastic contact triumph.

In many cases the classical friction model cannot capture the characteristics such as Stribeck effect, stick-slip, pre-sliding hysteretic motion, break-away force, which play a significant role in application on high precision positioning control (Abdo et al 2010, Abdo and Al-Yahmadi 2009). The role of friction modeling can be categorized according to whether or not the friction compensation is model-based. Examples of non-model-based compensators include high-gain feedback, and impulsive control.

This paper contains four sections including the introduction. Section 2 reviews the single state elastoplastic friction model given by Dupont et al. (2002). Section 3 presents an adaptive compensator to achieve velocity tracking in the presence of the friction force described by the single state elastoplastic friction model and a proof of it is also given in this section. 
Since the single state elastoplastic friction model contains a bounded function to describe different friction phases and depends on the immeasurable elastic state, the proposed compensator uses an adaptive observer and a computed force controller. Stability analysis of the proposed adaptive compensator is carried out using Lyapunov second method. Simulation results are given in Section 4 and conclusion is drawn in Section 5.

\section{Single State Elastoplastic Friction Model}

In this section we review the single state elastoplastic friction model given by Dupont et al. (2002), based on which we design an adaptive velocity compensator to follow a desired velocity trajectory. The rigid body displacement $x$ is composed of elastic $(z)$ and plastic $(w)$ components as,

$$
x=z+w
$$

Friction models define the elastic dynamics explicitly, while the plastic displacement $\mathrm{w}$ is defined implicitly. Following Dupont et al. (2002) model, the friction force is given by:

$$
\begin{aligned}
& f_{l}=\sigma_{o} z+\sigma_{1} \dot{z}+\sigma_{2} \dot{x} \sigma_{O}, \sigma, \sigma_{2}>0 \\
& \dot{z}=\dot{x}\left(1-\alpha(z, \dot{x}) \frac{z}{z_{s s}(\dot{x})}\right)
\end{aligned}
$$

where $z_{s s}$ is defined as

$$
z_{s S}=\left\{\begin{array}{c}
\frac{f_{S S}(\dot{x})}{\sigma_{o}} \quad|\dot{x}|>0 \\
\lim _{\dot{x} \rightarrow 0^{+}}\left(\frac{f_{S S}(\dot{x})}{\sigma_{o}}\right) \quad \dot{x}=0
\end{array}\right.
$$

and $f_{s s}(x)$ is the steady state friction force also called the Stribeck function which is shown in Fig. 1. The Stribeck function $f_{s S}(x)$ is bounded from below and above as

$$
f_{m} \leq f_{s S}(\dot{x}) \leq f_{M}
$$

and

$$
\alpha(z, \dot{x})=\left\{\begin{array}{lr}
0 & |z| \leq z_{b a}, \operatorname{sgn}(\dot{x})=\operatorname{sgn}(z) \\
\alpha_{m}(.) & z_{b a}<|z|<z_{s s}(\dot{x}), \operatorname{sgn}(\dot{x})=\operatorname{sgn}(z) \\
1 & |z| \geq z_{s s}(\dot{x}), \operatorname{sgn}(\dot{x})=\operatorname{sgn}(z) \\
0 & \operatorname{sgn}(\dot{x}) \neq \operatorname{sgn}(z)
\end{array}\right.
$$

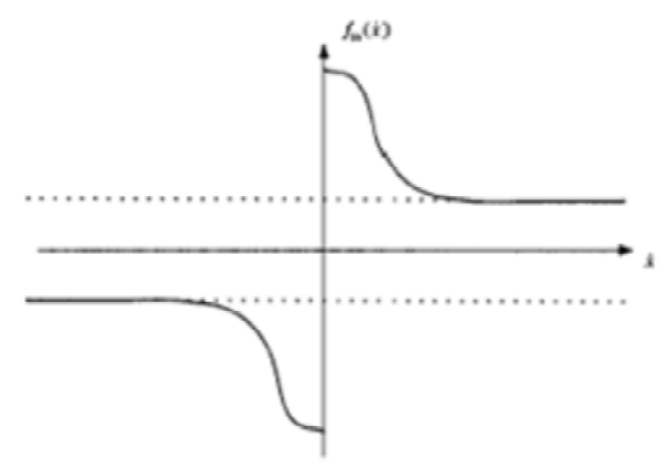

Figure 1. Stribeck curve of steady state friction force $f_{s s}(x)$ versus rigid body velocity

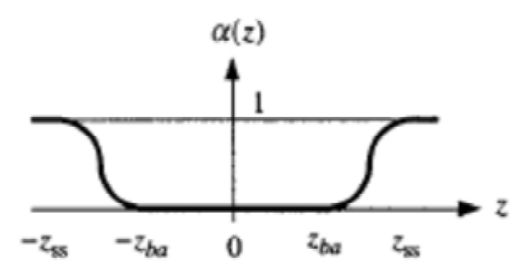

Figure 2 Plot of $\alpha_{m}(z, \dot{x})$ for $\operatorname{sgn}(\dot{x})=\operatorname{sgn}(z)$

which defines the elastic pre-sliding and mixed elastic and plastic displacements. Where $\alpha_{m}(z, \dot{x})$ is given as

$$
\begin{aligned}
\alpha_{m}(z, \dot{x}) & =\frac{1}{2} \sin \left(\pi \frac{z-\left(\frac{z_{s s}+z_{b a}}{2}\right)}{z_{s s}-z_{b a}}\right) \\
& +\frac{1}{2}, z_{b a}<|z|<z_{s s}(\dot{x})
\end{aligned}
$$

Figure 2 shows a typical shape of $\alpha_{m}(z, m)$ as given in (Dupont et al. 2002).

\section{Adaptive Compensator}

In this section an adaptive velocity compensator is designed to achieve tracking of a desired velocity trajectory $v_{d}(t)$ in the presence of friction force that was described by Eqs. (2 and 3). A similar approach was proposed by Canudas et al. (1995) but with a simple friction model and constant $\alpha_{m}(z, x)$. Consider the equation of motion of a single mass $m$ subject to driving force $\mathrm{u}$ and friction force $f l$

$$
m \dot{v}=u-f_{l}
$$

where $v$ is the velocity of the mass $(v=x)$.

Let $\bar{x}=(v z)^{T}$. The state space description of the complete system is composed of the rigid body model and the single state elastoplastic friction model is given as 


$$
\begin{aligned}
& \dot{\bar{x}}=\left(\begin{array}{cc}
-\frac{\sigma_{1}+\sigma_{2}}{m} & -\frac{\sigma_{o}}{m} \\
1 & 0
\end{array}\right) \bar{x} \\
& +\frac{\alpha(z, v)}{z_{s s}(v)}\left(\frac{\sigma_{1}}{m}\right) z v+\left(\begin{array}{c}
\frac{1}{m} \\
-1
\end{array}\right) u
\end{aligned}
$$

and in matrix form Eq. (9) can be given as

$$
\dot{\bar{x}}=A \bar{x}+\frac{\alpha(z, v)}{z_{s s}(v)} b z v+b_{c} u
$$

The only available signal for measurement is the velocity $v$ of the rigid body, hence, expressing $y=v$ in terms of the state $x$ leads to

$$
y=v=\left(\begin{array}{ll}
1 & 0
\end{array}\right) \bar{x}=c \bar{x}
$$

where

$$
\begin{gathered}
A=\left(\begin{array}{cc}
-\frac{\sigma_{1}+\sigma_{2}}{m} & -\frac{\sigma_{o}}{m} \\
1 & 0
\end{array}\right) \\
b=\left(\begin{array}{c}
\frac{\sigma_{1}}{m} \\
-1
\end{array}\right), \quad b_{c}=\left(\begin{array}{c}
\frac{1}{m} \\
0
\end{array}\right), c=\left(\begin{array}{ll}
1 & 0
\end{array}\right)
\end{gathered}
$$

Figure 3 shows the block diagram of the adaptive compensator, also known as state estimator, which consists of the rigid body with input force $u$, measured velocity $v$ and a state observer to compute an estimate of the state $\bar{x}$. The state model in Eq. (3) is numerically integrated to obtain the state estimator. The integrated state model is modified by adding additional terms to guarantee that the estimated state $\hat{\bar{x}}$ tends to the actual state $\bar{x}$. The block diagram of Fig. 3 shows also an adaptation law which calculates an estimate of the unknown function $\hat{\alpha}$ based on the estimated state $\hat{\bar{x}}$. The figure also includes a controller which is essentially a cancellation of the nonlinearities in the rigid body dynamics.

Since the state variable $z$ of the single state elastoplastic friction model is not measurable a state estimator is built to provide an estimate of $z$. $\hat{z}$ is a full order observer constructed to provide an estimate of $z$ and $v$ ( $\hat{v}$ is redundant). In addition, since the function $\alpha_{m}(z, \dot{x})$ depends on $z$, it is also unknown. A characteristic of $\alpha_{m}(z, \dot{x})$ is that it is bounded from below and from above (Dupont et al. 2002). We consider $\alpha_{m}(z, \dot{x})$ as an unknown parameter and construct an adaptive observer which provides an estimate of the unknown state $\bar{x}$ and the unknown parameter $\alpha_{m}(z, \dot{x})$. Since the model in Eq. (4) contains the product of two unknowns we use the Kaman-Yakubovich (KY) Lemma (Narendera and Annaswamy1989), which is based on the following definitions.

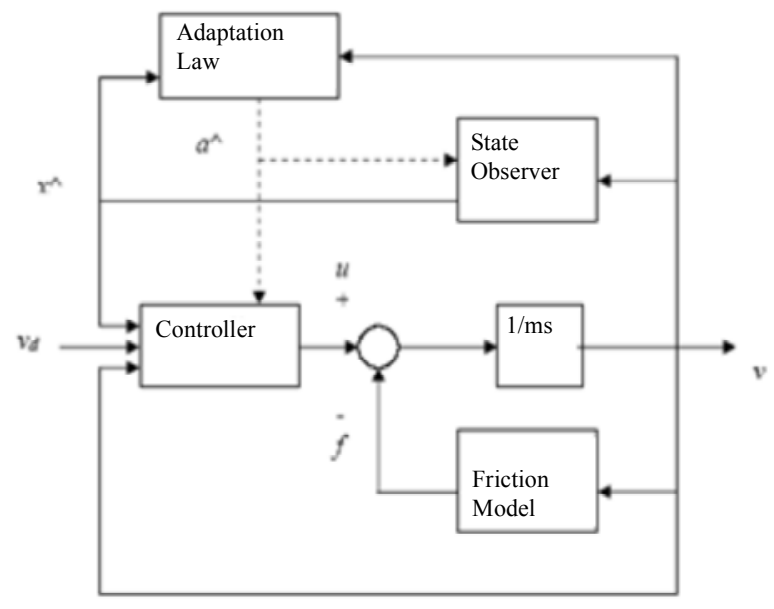

Figure 3. Adaptive Compensator

Definition 1 Positive Real (PR) function

A strictly proper rational function $G(s)$ is positive real (PR) if $G(s)$ is analytic for $\operatorname{Re}[s] \geq 0$ and $\operatorname{Re}$ $[G(j \omega)>0$ for al $-\infty<\omega<\infty$.

Definition 2 Strictly Positive Real (PR) function

A strictly proper rational function $G(s)$ is strictly positive real (SPR) if $G(s-\varepsilon)$ is positive real for some real $\varepsilon>0$.

The Kaman-Yakubovich (KY) Lemma (Narendera and Annaswamy 1989):

A strictly proper rational function $G(s)$ with state space realization $(A, b, c) G(s)=c(S I-A)^{-1}$ is strictly positive real (SPR) if there exists positive definite symmetric matrices $P=P^{T>0}$ and $Q=Q^{T>0}$, such that

$$
P A+A^{T} P=-Q
$$

\subsection{Adaptive Observer}

The adaptive observer is given as

$$
\begin{aligned}
\dot{\bar{x}} & =\left(\begin{array}{cc}
-\frac{\sigma_{1}+\sigma_{2}}{m} & -\frac{\sigma_{o}}{m} \\
1 & 0
\end{array}\right) \hat{\bar{x}} \\
& +\left(-k_{o} e_{o}+\frac{\hat{\alpha}(t)}{z_{s S}(\dot{x})} \hat{z} v\right)\left(\begin{array}{c}
\frac{\sigma_{1}}{m} \\
-1
\end{array}\right) \\
& +\left(\begin{array}{c}
1 \\
m \\
0
\end{array}\right) u+L(y-\hat{\bar{x}})
\end{aligned}
$$


where, $e_{O}=c(\bar{x}-\hat{\bar{x}})=c e, k_{O}>0$

$L=\left(\begin{array}{ll}l_{1} & l_{2}\end{array}\right)^{\mathrm{T}}$ is the observer gain and $l_{1}$ and $l_{2}$ are chosen to make the transfer function $G o(s)=c(s I-A+$ $L c)^{-1} b$ Strictly Positive Real (SPR). Since the pair $(c, A)$ is observable (Seabra and Berthe 1987) for $\sigma_{O} \pm 0$, we can place the eigenvalues of the matrix ( $A$ -

$L c)$ at any desired position. We notice also that the transfer function $G(s)=c(s I-A)^{-1} \mathrm{~b}$ has a zero at $\left(-\sigma_{\mathrm{o}}\right.$ $/ \sigma_{1}$ ) (transfer function equal to zero), hence by placing the eignevalues of the matrix $A-L c$ at the location of the zero $\left(-\sigma_{\mathrm{o}} / \sigma_{1}\right)$ the remaining eigenvalue is in the left half plane and thus we obtain a strictly positive real function (SPR). A zero of a transfer function is a real or complex number.

Depending on Kaman-Yakubovich $(K Y)$ Lemma there exist a positive definite symmetric matrices $P=$ $P^{T}>0$ and $Q=Q^{T}>0$, such that

$$
P(A-L c)+(A=-L C)^{T} P=-Q
$$

\subsection{Controller}

In order to steer the rigid body velocity, $v$, to follow a desired velocity, $v_{d}$, a control force $u$ is applied. The force consists of two parts: a cancellation of the nonlinear terms in the rigid body equation and a feedback term $v$ - $v_{d}$ to guarantee convergence of $v$ to $v_{d}$. To implement the first part the estimates $\hat{\bar{x}}$ and $\hat{\alpha}$ are obtained from the state estimator and the adaptation law are used.

The controller $u$ is chosen as

$$
\begin{aligned}
u & =m\left\{\dot{v}_{d}-k \widetilde{v}+\frac{\sigma_{1}+\sigma_{2}}{m} v\right. \\
& \left.+\frac{\sigma_{o}}{m} \hat{z}-\frac{\hat{\alpha}(t)}{z_{s s}(v)} \frac{\sigma_{1}}{m} \hat{z} v\right\}
\end{aligned}
$$

where $\tilde{v}=v-v_{d}, k>0$ and $\hat{\alpha}(t)$ is obtained from the adaptation law:

$$
\frac{d}{d t} \hat{\alpha}(t)=\gamma \frac{\left(e_{o}+\sigma_{1} \tilde{v} / m\right) \hat{z} v}{z_{s s}(v)}
$$

$\gamma>0$ is an adaptation gain.

\subsection{Stability Analysis}

The close loop system given by Eqs. (9), (14),(16) and (17) is analyzed in this section utilizing the Lyapunov Second Method. Consider

$$
e=\bar{x}-\hat{\bar{x}}, \widetilde{z}=z-\hat{z}=c_{1} e, c_{1}=(0
$$

hence

$$
\begin{gathered}
\dot{e}=(A-L c) e+\left(\frac{\alpha(z, v) z}{z_{s s}(v)}\right. \\
\left.v-\frac{\hat{\alpha}(t) \hat{z}}{z_{s s}(v)} v-k_{o} e_{o}\right) b
\end{gathered}
$$

From the selected gain $L$ discussed in section 3.1 to make the transfer function $c(s I-A+L c)^{-1} \mathrm{~b}$ SPR there exist a positive definite symmetric $P$ and $Q$ which satisfies Eq. (13).

Substituting for the control $u$ into (3.1) we obtain

$$
\begin{gathered}
\dot{\tilde{v}}=-k \tilde{v}+\frac{\sigma_{1}}{m}\left(\frac{\alpha(z, v) z}{z_{s s}(v)}-\frac{\hat{\alpha}(t) \hat{z}}{z_{s s}(v)}\right) \\
v-\frac{\sigma_{0}}{m} c_{1} e
\end{gathered}
$$

Consider the Lyapunov function

$$
W=e^{T} P e+\widetilde{v}^{2}+\frac{1}{\gamma}(\alpha-\hat{\alpha}(t))^{2}
$$

Time derivative of $W$ along-with the solution of Eqs. (16 and 17) is given as

$$
\begin{aligned}
& 2 e^{T} P b\left(\frac{\alpha(z, v) z}{z_{s s}(v)} v-\frac{\hat{\alpha}(t) \hat{z}}{z_{s s}(v)} v-k_{o} e_{o}\right) \\
& \quad+2 \frac{\sigma_{1}}{m} \widetilde{v}\left(\frac{\alpha(z, v) z}{z_{s s}(v)}-\frac{\hat{\alpha}(t) \hat{z}}{z_{s s}(v)}\right) v-2 \widetilde{v} \frac{\sigma_{0}}{m} c_{1} e
\end{aligned}
$$

where

$\lambda_{m}=$ minimum eigenvalue of $Q$, (and from $K Y$ lemma $e^{T} P b=e^{T} c^{T}=e_{O}$ and $\left\|c_{1}\right\|=1$ ), therefore:

$$
\begin{gathered}
\dot{W}=-e^{T} Q e-2 k \tilde{v}^{2}+\frac{2}{\gamma}(\alpha(z, v) \\
-\hat{\alpha}(t))(\dot{\alpha}-\dot{\hat{\alpha}}(t))+ \\
2 e^{T} P b\left(\frac{\alpha(z, v) z}{z_{s s}(v)} v-\frac{\hat{\alpha}(t) \hat{z}}{z_{s s}(v)} v-k_{o} e_{o}\right) \\
+2 \frac{\sigma_{1}}{m} \widetilde{v}\left(\frac{\alpha(z, v) z}{z_{s s}(v)}-\frac{\hat{\alpha}(t) \hat{z}}{z_{s s}(v)}\right) \\
v-2 \widetilde{v} \frac{\sigma_{0}}{m} c_{1} e
\end{gathered}
$$

In order to develop our elastoplastic friction model compensator, the assumption $\dot{\alpha} \approx 0$ is used. Since $\alpha(z, v)$ is bounded between 0 and 1 and the assumption that the variation of $\alpha(z, \dot{x})$ is slow, the elastoplastic friction model is developed so $\left|z_{s s}(v)\right| \geq \frac{f_{m}}{\sigma_{o}}$ hence 


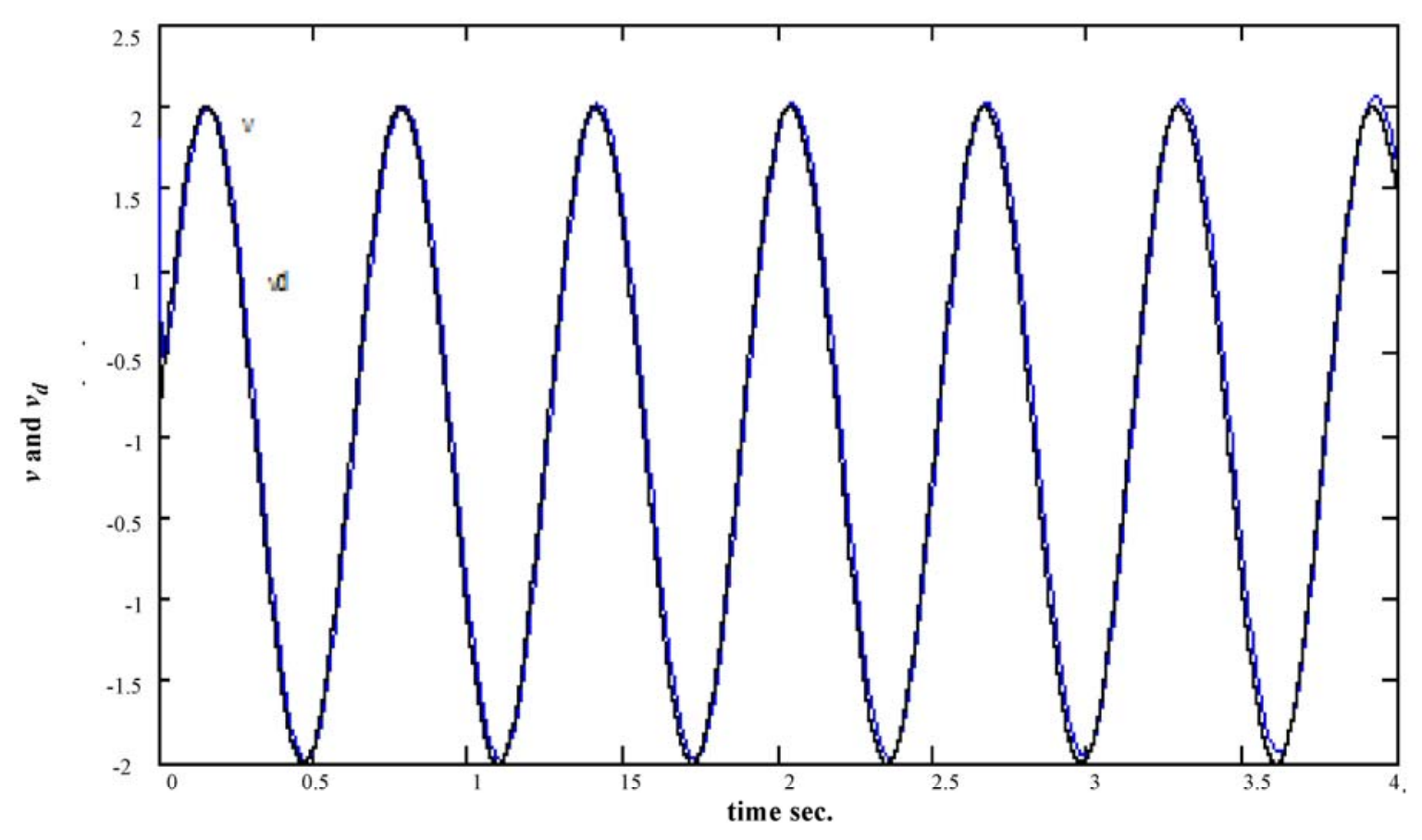

Figure 4. Rigid body velocity $v$ and desired velocity sinusoidal $v_{d}$

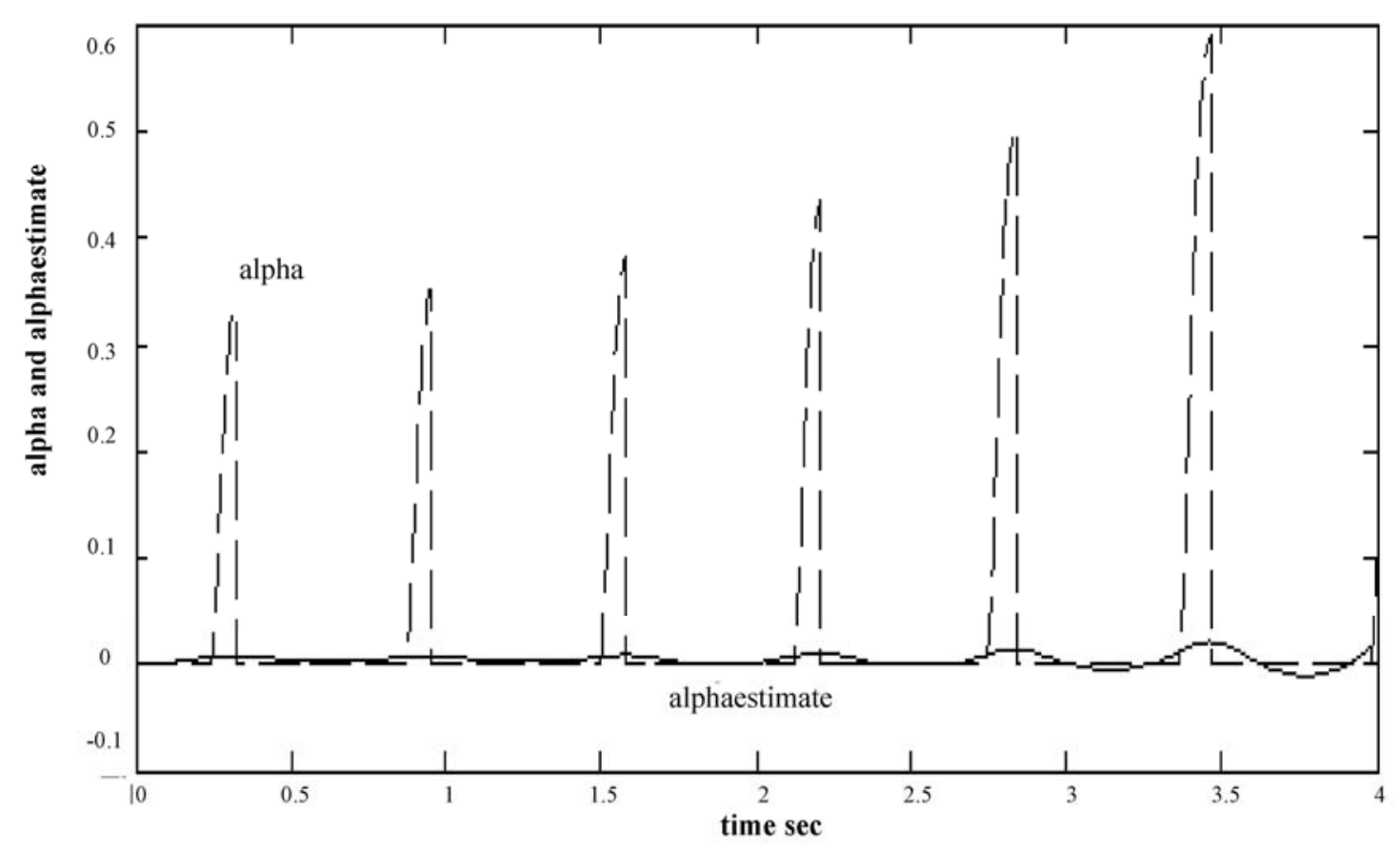

Figu re $5 \alpha(z, v)$ and $\hat{\alpha}(t)$ for sinusoidal desired velocity ("a" denotes $\alpha(z, v)$ and "estimate" denotes $\hat{\alpha}(t)$ ) 


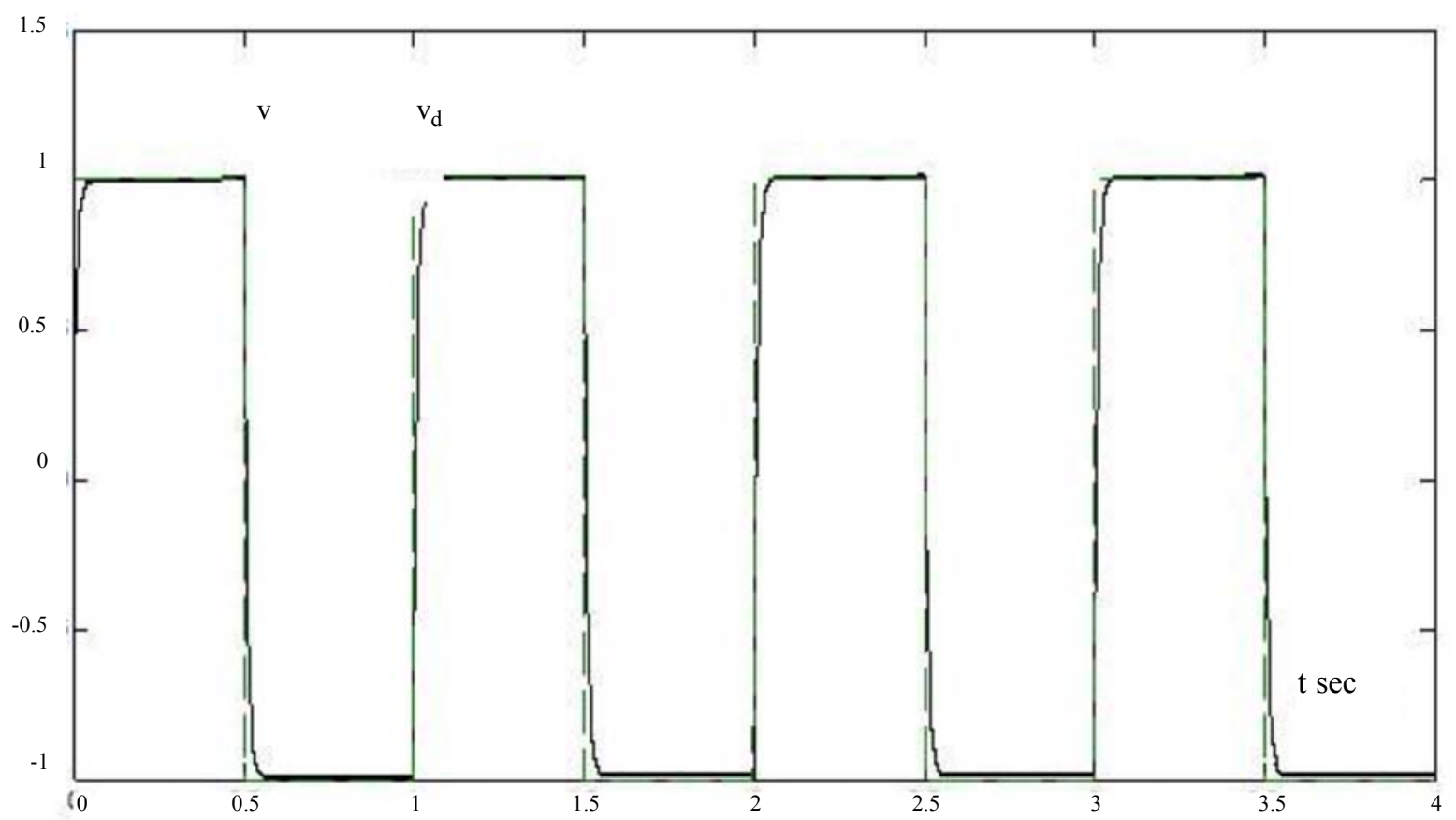

Figure 6. Rigid body velocity $v$ and desired square wave velocity $v_{d}$

Figure 6 shows the rigid body velocity which tracks almost accurately the desired square wave velocity. Figure 7 shows the estimates of $\alpha(z, v)$ and $\hat{\alpha}(t)$. It should be noted that the estimated value $\hat{\alpha}(t)$ (which is denoted by ae in the figure) is bounded. We attribute the non-converge of $\hat{\alpha}(t)$ to $\alpha(z, v)$ to the lake of persistency of excitation in the reference velocity $v_{d}$ (Narendera and Annaswamy 1989).

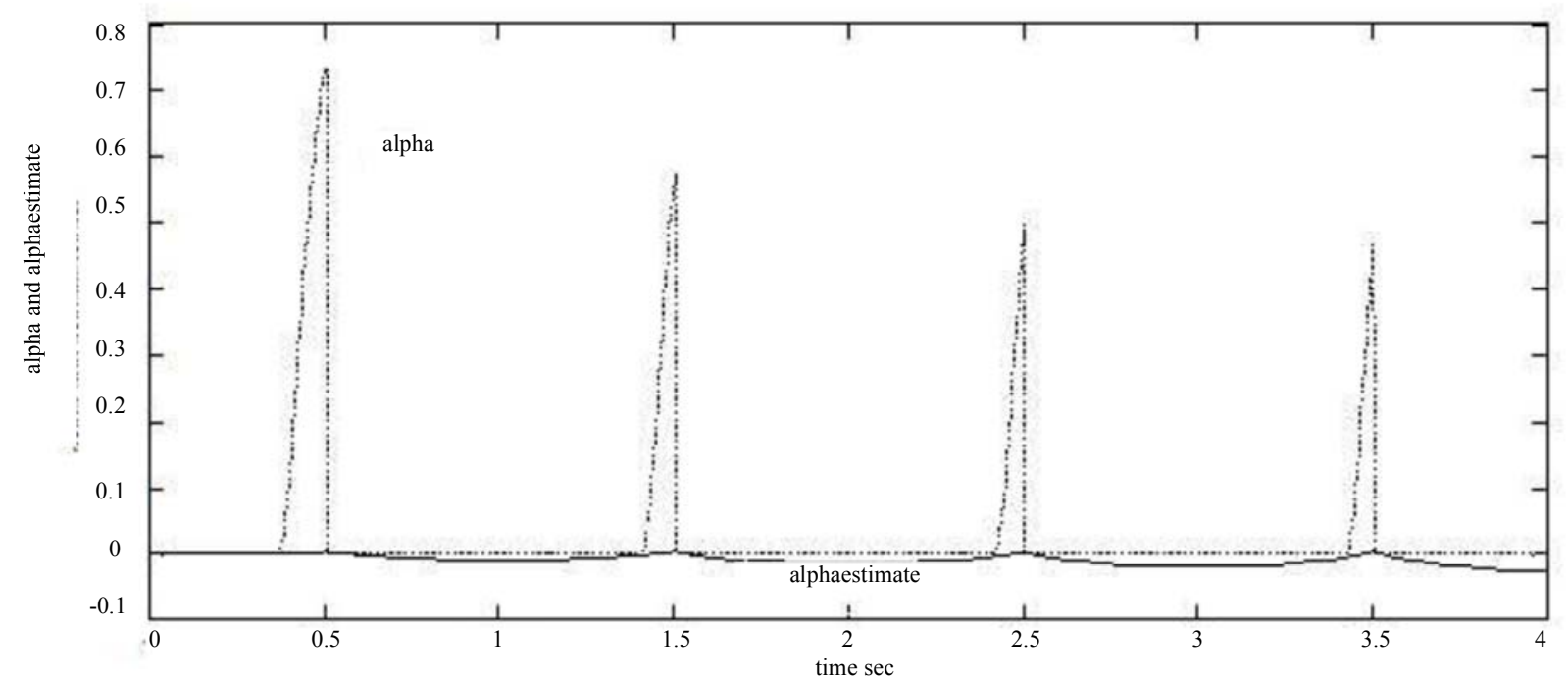

Figure 7. $\alpha(z, v)$ and $\hat{\alpha}(t)$ for square wave desired velocity (" $a$ " de not $\alpha(z, v)$ and "ae" denotes $\hat{\alpha}(t)$ ) 
This leads to

$$
\begin{aligned}
\dot{W} \leq & -\lambda_{m}|e|^{2}-2 k \tilde{v}^{2}+2\left|e_{o}\right| \frac{\sigma_{O}}{f_{m}}\|e\| v \mid \\
& +2 \frac{\sigma_{1}}{m}|\tilde{v}| \frac{\sigma_{O}}{f_{m}}\|e\|\left\|v\left|-2 k_{o} e_{o}^{2}+2 \frac{\sigma_{O}}{m}\right| \widetilde{v}\right\| e \|
\end{aligned}
$$

Choosing $\dot{\hat{\alpha}}(t)=\gamma \frac{\left(e_{O}+\sigma \tilde{v} / m\right) \hat{z}}{z_{S S}(\dot{x})} v$, Eqn. (21) can be rewritten as

$$
\begin{aligned}
\dot{W} \leq & -\lambda_{m}|e|^{2}-2 k \tilde{v}^{2}+2\left|e_{o}\right| \frac{\sigma_{O}}{f_{m}}\|e\| v \mid \\
& +2 \frac{\sigma_{1}}{m}|\widetilde{v}| \frac{\sigma_{O}}{f_{m}}\|e\|\left\|v\left|-2 k_{o} e_{o}^{2}+2 \frac{\sigma_{O}}{m}\right| \widetilde{v}\right\| e \|
\end{aligned}
$$

or

$$
\begin{aligned}
\dot{W} \leq & -\frac{1}{2} \lambda_{m}|e|^{2}-k \tilde{v}^{2}-\left(\frac{1}{4} \lambda_{m}|e|^{2}\right. \\
& \left.-2\left|e_{o}\right| \frac{\sigma_{O}}{f_{m}}\|e\| v+2 k_{o} e_{o}^{2}\right\}-\left\{\frac{1}{4} \lambda_{m}|e|^{2}\right. \\
& \left.-2\left[\frac{\sigma_{1}}{m} \frac{\sigma_{O}}{f_{m}}|v|+\frac{\sigma_{O}}{m}\right] \mid \tilde{v}\|e\|+k v^{2}\right\}
\end{aligned}
$$

To regulate the velocity $v$ to follow $v_{d}$ we assume $|v|<V_{M}$. By completing the squares the following inequalities are reached

$$
k_{o}>\frac{\left(\frac{\sigma_{o}}{f_{m}} V_{M}\right)^{2}}{0.5 \lambda_{m}} \text { and } k>\frac{\left(\frac{\sigma_{1}}{m} \frac{\sigma_{o}}{f_{m}} V_{M}+\frac{\sigma_{o}}{m}\right)^{2}}{0.25 \lambda_{m}} .
$$

These inequalities are used to satisfy $\dot{W} \leq-\frac{1}{2} \lambda_{m}|e|^{2}-k \widetilde{v}^{2} \leq 0$. Hence all trajectories are bounded and the origin is stable. Differentiating $\dot{W}$ with respect to time, $\ddot{W}$, leads to bounded inequality. Using Barbalat Lemma (Narendera and Annaswamy, 1989) we have $e, \widetilde{v} \rightarrow 0$ as $t \rightarrow \infty$. Thus the system velocity $v$ tracks the desired velocity $v_{d}$.

\section{Simulation Results}

The desired velocity function is chosen as $v_{d}(t)=$ $10 \sin (10 t)$ this is a standard test signal. The elastoplastic friction model parameters are given in Table 1 .

The Stribeck friction function is given by:

$$
\begin{aligned}
& f_{s s}=\left[f_{m}+\left(f_{M}-f_{m}\right) e^{-(1000 \mathrm{v}) 2}\right] \operatorname{sign}(v) \\
& z_{b a}=0.7169 Z_{s s}
\end{aligned}
$$

The gains of the observer and controller are given in Table 2.

It is obvious from Figures 4-7 that the proposed adaptive compensator provides an accurate tracking
Table 1. System parameters

\begin{tabular}{llllcl}
\hline $\mathrm{M}$ & $\sigma_{o}$ & $\sigma_{1}$ & $\sigma_{2}$ & $\boldsymbol{f}_{m}$ & $\boldsymbol{f}_{M}$ \\
\hline $10 \mathrm{~kg}$ & $100 \mathrm{~N} / \mathrm{m}$ & $10 \mathrm{Ns} / \mathrm{m}$ & $0.4 \mathrm{Ns} / \mathrm{m}$ & 50 & 200 \\
\hline
\end{tabular}

Table 2. Compensator gains

\begin{tabular}{cccc}
\hline$L$ & $\mathrm{~K}$ & $k_{o}$ & $\gamma$ \\
$(13.960 .6667)^{\mathrm{T}}$ & 100 & 10 & 0.1 \\
\hline
\end{tabular}

capability to the desired trajectory while keeping all other signals bounded.

Figure 4 shows the rigid body velocity which accurately tracks the desired velocity $v_{d}(t)=10 \sin (10 t) . \quad \alpha(z, v)$ and $\hat{\alpha}(t)$ for sinusoidal desired velocity are shown in Fig. 5.

\section{Conclusions}

In this paper a single state elastoplastic friction model is reviewed and an adaptive compensator is proposed to track a desired velocity trajectory in the presence of friction force model. The adaptive compensator includes an adaptive observer which consists of a state estimator and an adaptation law to one of the unknown functions of the friction model. The controller cancels the nonlinearities in the friction model using the estimated state and parameter. The model stability is determined by using Lyapunov analysis. Simulation of the proposed adaptive compensator to track both sinusoidal and square wave signals show almost perfect tracking and signals are bounded in the closed loop system which indicate the proposed compensator is effective.

\section{References}

Abdo J, Al-Yhmadi A (2009), The effect of controlled frequency and amplitude of vibration on friction. Solid state phenomena 147:380-385.

Abdo J, AL-Yahmadi A (2004), A wear model for rough surfaces based on the ultimate-stress asperity concept. Int. J. of Applied Mechanics and Engineering 9:11-19.

Abdo J, Farhang K (2005), Elastic-plastic contact model for rough surfaces based on plastic Asperity concept. Int. J. of Non-Linear Mechanics 40/4: 495-506.

Abdo J, Tahat M, Abualsoud A, Danish M (2010), The effect of frequency of vibration and humidity on the stick-slip amplitude. Int. J. of mechanics and materials in Design 6(1):45-51.

Canudas C de Wit, Olsson H, Astrom KJ, Lischinsky $\mathrm{P}$ (1995), A new model for control of system with 
friction. IEEE Transaction in Automatic Control 40(3):419-425.

Dupont P, Hayward V, Armstrong B, Altpeter F (2002), Single state elastoplastic friction models. IEEE Transaction in Automatic Control 47(5):783-787.

Greenwood JA, Williamson JBP (1966), Contact of nominally flat surfaces. Proc. R. Soc. 295:300.

Greenwood JA, Tripp JH (1971), The contact of two rough nominally flat rough surfaces. Proc. Instn. Mech. Engrs. 185:625-633.

Halling J, Nuri KA (1972), Contact of rough surfaces of working-hardening materials. In The Mechanics of Contact between Deformable Bodies, Eds. De Pater and Kalker. Delft: University Press 412.
Ibrahim RA, Rivin E (1994), Friction-induced vibration, Part I: mechanics of contact and friction. Applied Mechanics Reviews 47:209-226.

Mulhearn TO, Samuels LE (1962), The abrasion of metal: A model of the process, wear 5:478-498.

Narendera KS, Annaswamy AM (1989), Stable adaptive systems Prentice Hall.

Pullen J, Williamson JBP (1972), On the plastic contact of rough surfaces. Proc. Roy. Soc (London) A327:159-173.

Seabra J, Berthe D (1987), Influence of surface waviness and roughness on the normal pressure distribution in the hertzian contact. ASME J. of Tribology 109:462-470. 